\title{
Asset management and maintenance: a smart grid perspective
}

\author{
Chan-Mook Jung ${ }^{1}$, Papia Ray $^{2}$, Surender Reddy Salkuti ${ }^{3}$ \\ ${ }^{1}$ Department of Railroad and Civil Engineering, Woosong University, Republic of Korea \\ ${ }^{2}$ Department of Electrical Engineering, Veer Surendra Sai University of Technology, India \\ ${ }^{3}$ Department of Railroad and Electrical Engineering, Woosong University, Republic of Korea
}

\begin{tabular}{l} 
Article Info \\
\hline Article history: \\
Received Mar 1, 2019 \\
Revised Apr 3, 2019 \\
Accepted Apr 12, 2019 \\
\hline Keywords: \\
Aging assets \\
Asset management \\
Renewable energy resources \\
Smart grid \\
Uncertainty
\end{tabular}

\begin{abstract}
This paper presents the importance, issues and challenges related to Smart Grid. It also evaluates various approaches for Smart Grid planning and operation. It discusses tools for asset management and their applicability to the next generation grid. Aging assets, uncertainty in load demand profile and renewable energy resources, and demand management create a challenge for the optimal operation and maintenance of electrical grid. This paper addresses the challenges and opportunities to improve transmission and distribution systems asset maintenance. This paper also presents the asset replacement alternatives. This paper also presents the cost-benefit analysis of asset management using the information/real time data from the utility company. This paper will serve a guide for doing the asset management to the electrification process, investment and recovery to sustain reliable and efficient power delivery.
\end{abstract}

Copyright $@ 2019$ Institute of Advanced Engineering and Science. All rights reserved.

\section{Corresponding Author:}

Surender Reddy Salkuti,

Department of Railroad and Electrical Engineering,

Woosong University,

17-2, Jayang-Dong, Dong-Gu, Daejeon 34606, Republic of Korea.

Email: salkuti.surenderreddy@gmail.com

\section{INTRODUCTION}

Asset Management (AM) is one of the key issue in the Smart Grid context. Aging assets and uncertainty in load demand profile and demand management create a challenge for the optimal operation and maintenance of electrical grid. The proper asset management reduces the risk of equipment failure, extends the equipment life and minimizes the occurrence of unplanned outages. AM refers to any system where the things that are of value to an entity are maintained and monitored. AM is the systematic process of maintaining, operating and upgrading assets with minimum possible cost [1]. Enterprise AM (EAM) is the business processes and enabling information systems that support management of an organization's assets. Physical AM (PAM) is the practice of managing the whole life cycle of physical and infrastructure assets such as production and service plant, power, structures, water and waste treatment facilities, transport systems, distribution networks, buildings and other physical assets. Infrastructure AM expands on this theme in relation primarily to public sector, utilities and transport systems.

Background: Fixed AM (FAM) is the process of accounting which offers to track fixed assets for the financial accounting purposes. IT asset management (ITAM) is the set of business practices which joins the contractual, financial and inventory functions to strategic decision making and support life cycle management for the IT environment [2]. Digital asset management (DAM) is a form of electronic media content management which includes the digital assets. Power systems face several new challenges which includes the sheer complexity that results from the introduction of new devices like phasor measurement units (PMUs), Geographical Information Systems (GIS), sensors and advanced controllers on equipment throughout the system, intelligent electronic devices (IEDs) in substations, electric and hybrid vehicles, smart meters, distributed storage systems, photovoltaic generation and wind generating units [3]. In [4], an application- 
based distribution network asset management is discussed by proposing a dynamic network rating methodology to facilitate the low carbon network operation. A two-step approach for the scheduling of an electric vehicle (EV) charging while limiting the burden on transmission and distribution assets is proposed in [5].

The Problem: Reference [6] develops a model for determining the location and number of automatic switches in distribution networks with the objective of minimizing the investment, operation, and unreliability costs. In Reference [7], an intelligent framework for the condition monitoring and the assessment of power transformers is proposed while using the data from historic database and online sensor measurement. Multi-objective decision-making condition based maintenance model is proposed in [8]. A cascading failure risk assessment method based on the reliabilities model of circuit breakers and protective relays is proposed in [9], where a fully physical mechanism is utilized to illustrate the evolution of cascading failures. Asset Management can be improved in presence of these new devices, new control strategy, new communication equipment and new sensors in the system. The performance-based AM (PAM) is an important tool which can be used in coordination with the fundamental technologies of smart grid: sensing and measurement, integrated communications, improved interfaces and advanced control approaches with decision support [10].

Various asset management strategies in the distribution networks based on short-term, mid-term and long-term time scales are proposed in [11]. Reference [12] asserts that the realization of chronological AM in the field could lead to the better management and operation of power systems in the competitive era. The use of more sophisticated control technologies and algorithms such as inference engines, expert systems, knowledge bases, and other advanced processing methods have been studied for many years. Researchers have diverted to new methodologies for optimization such as Adaptive Dynamic Programming (ADP), evolutionary programming and heuristic programming. The methods available in the literature such as Fuzzy Logic, Stochastic Programming and Mixed Integer Programming, etc. are also improvised to fit to the new environment [13]. The theory of life cycle AM within the cutting-edge technology in Smart Grid (SG) is analyzed in [14], and a complete and mutual management method is also presented.

The Proposed Solution: Frequent condition assessment with asset audit enables a comprehensive assessment of the asset in question and includes objective and transparent asset condition information recommendations for the improved environmental sustainability. Based on the individual maintenance schedules or on the assessment and inspection findings, maintenance operations are planned to ensure the reliability of the system. With penetration of Renewable Energy Resources (RERs), grid parameters involve stochasticity and randomness. Moreover, load pattern is always random and unpredictable. So, with this new environment Smart Grid have to sustain its operation efficiently [15].

It is possible only with new load flow and optimal power flow techniques. So, the new power flow and optimal power flow should be dynamic and it should be applicable to real time applications. So, the methodology suggested by various researchers is directed towards next generation optimization techniques such as Adaptive Dynamic Programming (ADP), Stochastic Programming (SP), Mixed Integer Programming (MIP), Evolutionary Programming (EP) and Heuristic Programming, etc. These methods are capable of solving the randomness involved into the system. As the utility industry concentrates on the complex and ambitious transformation of the outdated power grid to the new smart grid, it has struggled to develop a shared vision for the smart grid end-state and the path to its development and deployment. Now, the smart grid maturity model (SGMM) is helping the industry to overcome these challenges by presenting a consensus vision of smart grid, the benefits it can bring and various levels of smart grid development and deployment maturity. SGMM is helping numerous utilities worldwide to develop targets for their smart grid strategy, and build roadmaps of activities, investments and best practices that will lead them to their future smart grid state. Today, the biggest hurdle we face regarding the substations is that utilities cannot schedule outages because of the load and demand on their substations.

In the past, the systems were built to take on extra load and utilities planned on having sufficient extra margins to allow other parts of the system to take over. But, now to reduce the costs, systems are managed with lower margins, therefore this approach cannot be applied any more. On-line asset monitoring can be used to point out the abnormal stresses and the ageing of a component, before a failure occurs, and to obtain data essential for the planning of suitable maintenance measures. This operation can be carried out in a period when the unavailability of that particular part of the system does not impair the overall system operation, i.e., when other redundant part can guarantee the operation. Under these situations the system achieves an interruption-free diagnostic. The aim of this paper is to analyze the asset management and maintenance of smart grids. This paper also presents the challenges and improvements required to match the grid standards and expectations. 
The remainder of the paper is organized as follows: Section 2 describes the asset management in Smart Grid. The tools for the asset management are presented in Section 3. Finally, the contributions with concluding remarks is presented in Section 4.

\section{ASSET MANAGEMENT IN SMART GRID}

Efficient management of utility assets has been a priority for the utilities. As a means of improving the profitability and reliability taking care to build and maintain plant at an appropriate level of investment and quality [12]. Balancing operating expenses and capital costs to deliver the best value for utility customers and shareholders is the major objective of asset management. The task has grown in complexity, with the spiraling cost of new and replacement plant. Most utilities use some form of automation to address the problem, tailored to the unique business processes of building and maintaining plant. With the advent of Smart Grid (SG) technology adds at least two dimensions to the asset management (AM) problem. First, there are number of new types of monitoring and control devices that are or will be added to the network, from substation and line sensors to smart meters [16]. The asset models of many utilities will have to be expanded to add these new devices, while the some existing assets, like smart switches, will change and improvised to fit to the new environment. Second, the definition of a critical asset will evolve, and will undoubtedly grow to include more of the distribution grid [2].

The legacy approach to AM through the use of diverse, multiple, synchronized databases and interfaced systems will likely be unable to manage the requirements placed on utilities to operate their distribution systems in a smarter way. In the dynamic smart grid, it will no longer feasible to rely on distributed technology systems with large numbers of interfaces, or to have multiple instances of assets that require constant synchronization. To streamline the asset management process, utilities should work towards achieving specialized asset-intensive software applications. Establishing a single instance of an asset and managing updates through a unified process is the goal of this vision [3].

The elimination of use of "special purpose" databases for SG assets, the utilities should consider focusing in on a single asset platform. As an example, many utilities use same AM software for power equipment. With some work, that application could be implemented to manage the additional assets that come along with the SG era, including communications equipments, smart devices and the equipments required to manage the distributed generation. Consolidation will not be pain free, but will reduce the number of systems involving the asset management, streamline the business processes, and greatly simplify the Information Technology (IT) environment [17]. Advanced Meter Infrastructure (AMI) data gives the utility information to unlock greater value. This information is only available and usable, however, if the utility has a fully functional and accessible data store. This requirement has been a key driver behind many Meter Data Management Systems (MDMS) implementations as part of smart metering initiatives [15]. Currently available strategy for operations and planning has following limitations:

a. Voltage control is achieved with devices such as capacitor banks, voltage regulators and Tap Changing Transformers which have localized controls. These schemes work well for today's radial circuits but they do not handle the circuit reconfigurations and voltage impacts of local generation well, resulting in limits on the ways in which circuits can be configured and imposing the important limits on the penetration of distributed resources. Sometimes these local controls also invite major blackouts.

b. Minimal metering and communication infrastructure is in place to aid in restoration following faults on the system.

c. No effective communication infrastructure exists to facilitate management and control of distributed resources that could include renewables, other distributed generation, and storage.

d. There is no communication to customer facilities to allow customer loads and customers to react to emergency conditions, electricity price changes, or both. Customer-owned and distributed resources cannot participate in electricity markets, limiting the economic payback in many cases.

The information provided covers not only interval energy usage, but also status, events and alarms. Even utilities in regions with a central MDMS deployment (e.g., a single MDMS implementation for the entire regulatory jurisdiction such as a state, province or country that provides a common data repository, bill determinant calculations and customer usage presentment for all utilities and customers in the jurisdiction) have benefited from the operational visibility and efficiencies achieved from their own smart metering infrastructure data [18]. One such benefit is the ability to monitor and act on health and tamper information reported by smart meters. Enabling such functionality for all utilities would typically be beyond the scope of a central MDMS implementation [16]. An MDMS provides separation of concerns in a utility's applications architecture. The MDMS can handle all the responsibilities related to meter data and can serve as a one-stop point for all current and historical meter usage information, thereby establishing the consistent 
processes for publishing meter data to users of information within the enterprise. The overview of Asset Management (AM) processes are described in Figure 1.

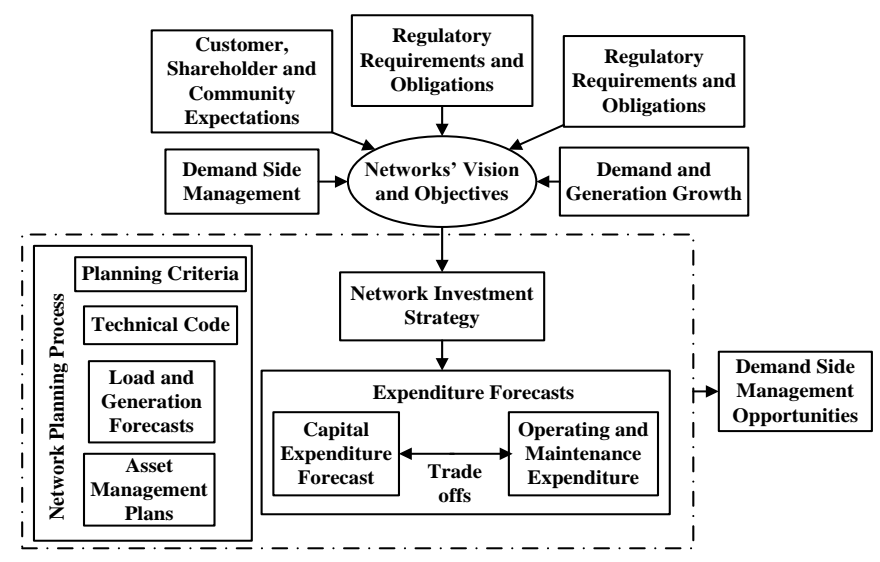

Figure 1. Overview of Asset Management (AM) processes

In addition to managing the energy metering data for smart meters, an MDMS can also provide a collection of adapters and interfaces to integrate multiple smart metering infrastructure systems that use different technologies and data formats. This feature effectively decouples the downstream applications from the automated metering infrastructure, allowing the integration of new technologies as they emerge, and the decommissioning of old technologies, without being restricted to a single vendor or AMI implementation [19]. This means consumers of AMI data outputs can use the MDMS as a single, consistent interface across various AMI systems, with data presented in a standardized manner. Collection management refers to the ability of an MDMS to provide sophisticated capabilities such as read arbitration, read integrity inspection, data rejection, data aggregation, scheduling and service level agreement (SLA) monitoring across multiple smart metering infrastructure head-ends and systems. This is a key benefit of MDMS implementations in terms of technical scoping, particularly where utilities expect to deploy multiple smart meter systems to cover their territory. Figure 2 depicts the condition monitoring using the balanced approach [20].

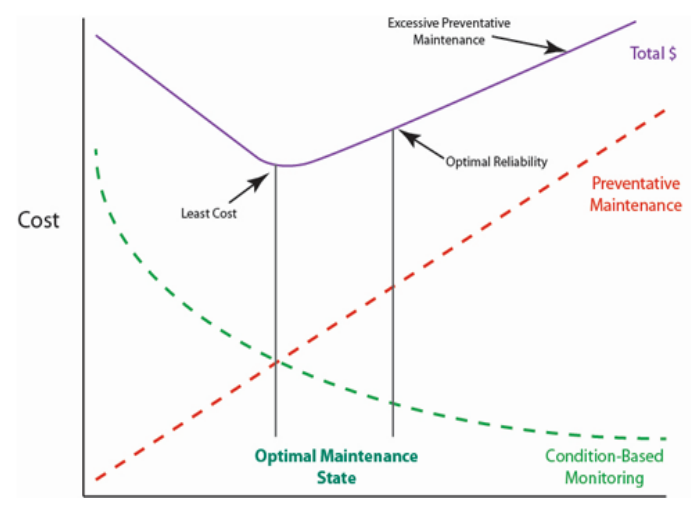

Figure 2. Condition monitoring using balanced approach

The Power industry's knowledge and experience of SG benefits and capabilities are growing rapidly, and the utilities' SG plans are becoming more sophisticated and advanced every day. AMI was considered as the useful tool for the SG, but now the utilities realize that the Distribution Management System (DMS) is the most important components of an effective SG strategy [21]. Accurate data is required for the DSM. The DMS based on network model must accurately reflect the real time data, transactional data, time series data and the network asset information. GIS is very important in the network data integrity. GIS data has crucial role in SG to maintain the up-to-date and accurate network model that must be fed to all SG 
information systems, such as Supervisory Control and Data Acquisition (SCADA), Distribution Managements System (DMS), Meter Data Management (MDM), Outage Management System (OMS), and others [22].

Modern power grids are continuously monitored by the well-trained system operators (SOs) equipped with the sophisticated control and monitoring systems. Despite of such precautionary measures, major blackouts still occur quite often around the globe. To prevent these blackouts, it is very important to perform higher-order contingency analysis [23]. However, such contingency analysis is computational burden, because several possible combinations of power system component (lines, transformers, and generators) failures must be analyzed. Moreover, with new grid the list of contingencies may expand as well. This paper involves the development of parallel probabilistic contingency analysis algorithms and load balancing schemes that are scalable and achieve near optimal allocation of computational tasks to processors. It can be done with next generation optimization tools like Adaptive Dynamic Programming (ADP), Evolutionary Programming, Heuristic Programming, Stochastic Programming, Adaptive Fuzzy Logic, Mixed Integer Programming, etc.

The dynamic voltage stability indices can be developed with the help of Phasor Measurement Unit (PMU). The PMU provides real time information about voltage and phase Angle at every 30 seconds through a waveform generation. This waveform can be converted into real numbers with the help of pi systems. OSIsoft is one of the software that converts this signature information into number. Achieved numbers can also be verified through the state estimator (SE) and it will eliminate the bad data if any present in the system. Hence, the achieved numbers now can be used for voltage stability indices calculation [24]. In case we don't have a PMU installed, we can use any optimization tool to generate data such as Artificial Neural Network (ANN), Partical Swarm Optimization (PSO), Ant Colony Optimization (ACO), etc. to create the missing numbers through Artificial Intelligence (AI), Evolutionary Programming (EP) or Heuristic Programming. After the numbers generated, we can use this numbers for the calculation of Voltage Stability Indices (VSI). The generated VSI will be dynamic and gives real time information about the voltage stability. It's value varies between 0 and 1 . If the value is close to one, then the system is more stable. Therefore, it gives the measure of how far is the system from instability. A fuzzy logic based index has been proposed in the literature and is applicable in real time as well [22].

\section{TOOLS FOR ASSET MANAGEMENT}

Asset owners utilizes various software tools to manage their utility plants are described in this section. Each of these software tools has different business application, although some overlap in functionality [21]:

a. Asset/Work Management Systems - These systems concentrate on record keeping and asset lifecycle tracking, including the work resources applied to construct and maintain the plant [25].

b. Fixed Asset Accounting - Fixed asset systems manage the property records of the business from a financial value standpoint.

c. Geographical Information System (GIS) - These systems are used to design new facilities, model the relationships of assets to the electrical network, and track as-built assets through a lifecycle generate maps and sketches.

d. SCADA - These systems maintain sufficient asset information to enable the dispatchers to operate the network in "real-time".

e. Planning Systems - Utilities use various planning software solutions to model the network and analyze its performance under existing and projected load conditions.

f. DMS - Combining the network model and analytics of planning tool with the real-time infrastructure and control of SCADA system, DMS allows system operators to make more intelligent operational decisions, or in some cases, makes those decisions automatically.

The use of distributed systems often leads to sub-optimal asset performance. Generally, the legacy systems are implemented independently, and by different parts of the utility, to meet different business needs. Assets tend to be replicated in many systems, leading to a host of gaps, errors, and inefficiencies [26]. Asset owners have responded with various solutions, including integrating various IT systems that model assets, or implementing a cross cutting enterprise resource planning system (ERP) in an attempt to develop a single system of record for the asset. Some worked well, and others well enough, and still others, not so well at all. But, as the Smart Grid era approaches, pressure to improve even the best asset management practice will only intensify.

Smart Grid concept is really young and it came into main focus only a few years ago. At the highest level, the most important drivers motivating a more intelligent grid include [27]: 
a. Changing Demand Patterns - Growth in electrical energy use, from computer loads to electric vehicles, to generally increasing reliance on electric energy has put pressure on all aspects of the grid. Many utility customers are seeking to know more about, and even to control, their electrical energy usage.

b. Higher reliability requirements - As the cost of outages and poor service increase, even the most reliability focused utilities are concentrating on improving or maintaining performance. To enable greater visibility, additional telemetry devices are being deployed on the distribution network. There is a strong movement to add intelligent switching to the distribution systems to support "self-healing" operations, to balance load, and to resolve the outages quickly.

c. Distributed Generation - Distributed, "green" generation is growing rapidly throughout the world as a result of environmental concerns, advancing technology, and economics. This adds more complex assets to the distribution system, and creates the challenges around operating those assets in a sustainable and reliable manner.

d. Operating efficiencies are even more attractive - As the cost for new plant increases, and the political and economic environments make more generation capacity less viable, utilities are seeking to squeeze ever more performance out of the existing plant. With minimal upgrades, adding more sophisticated analysis and control can enable utilities to operate within the narrow parameters, optimizing use the of network assets.

These drivers imply that a large number of new distribution assets will be added, including the substation, feeder, end-point devices, and telecommunications infrastructure that transmits real and near real time data to the utility [28]. The result is a rapid growth in additional assets in the distribution system with ancillary attributes which are not generally seen before. Added to volume is the increased frequency of plant changes in the network, straining the ability of traditional asset management (AM) tools. The utility industry has undertaken the next evolutionary phases of Smart Grid development, in which a new generation of hardware and software Advanced Grid Management (AGM) is enabling merger and multiple rapid analyses of new Smart Grid data streams. Utilities have started using the new analytic results for advanced business and planning applications, real-time visualization, increased automation, improved asset management, and soon real-time grid operations.

In response to new challenges, distribution utilities continue developing a smarter grid. They have supplemented Energy Management Systems (EMS) and SCADA already in place by deploying new systems such as GIS applications, Meter Data Management Systems (MDMS), Advanced Metering Infrastructure (AMI), automated Outage Management Systems (OMS), Demand Response Management Systems (DRMS), and Distribution Management Systems (DMS) [29]. These systems represent the three initial evolutionary phases of the still-evolving smart grid [30]:

- Installation of intelligent hardware devices.

- Connection and interconnection of the devices via enterprise communication broadband networks for fast data transmission.

- Integration of data with open-architecture utility IT systems via newly standardized open architecture and interoperability requirements, to facilitate communication, processing, sharing, and analysis of new data streams.

This will allow utilities to accurately prioritize asset and load management, refine demand response, storage, generation dispatch, and fulfill regulatory mandates for renewable energy portfolio integration, energy efficiency, customer service and demand response [28]. Smart Grid (SG) is also lifeblood of real time measurements involved in the smart grid. It allows two way communication and many researchers have discussed in real time pricing by either taking access of consumer energy consuming equipment or by spread awareness about peak time usage and its disadvantages. The former way is controversial and it may not be applicable in practical but at least a later way allows dynamic pricing to be applicable. Geographical Information System (GIS) is the other real time application that can help in measurement and control of smart grid. The information obtained through GIS can be extended to fault centers and the physical faults can be minimized and solved very quickly. Therefore, the GIS can help to maintain reliability of the system.

As the smart grid technologies began to be deployed rapidly during the past decade in the predominant forms of AMI, distribution automation techniques and control room software like DMS, few companies understood that adding those assets to a companywide AM strategy portfolio is very important. AM involves prioritizing and optimizing investments in assets to improve or to maintain performance and life expectancy throughout the asset's life cycle. Smart AM (SAM) uses the smart grid (SG) data to improve AM results through the use of data analytics and back-end integration [24]. AM applications are heavily data driven requiring data from across the enterprise from GIS, the maintenance management system, work management system, on-line monitoring system, outage management system, equipment catalogs, standards, meter data management systems, planning and archived operational histories. The advantages of implementing the smart asset management include cost savings and a better understanding 
of risk; improved performance from existing equipment; asset life cycle and procurement planning; a defendable, vigorous, repeatable platform for justification of asset decisions with advanced auditing capability; compliance with regulations and improved customer satisfaction through better reliability and power delivery; improved system reliability with increased longevity of asset life by maintaining operation within asset specifications.

The smart grid has the potential to change how the industry approaches AM with the adoption and integration of advanced information collection, communication systems, and control devices, and software to economically deliver the optimal functionality [30]. Intelligent and advanced AM can be implemented through an efficient connection of mobile, local server and cloud based systems. Connect to time dynamic external factors and convey the best management strategies to the enterprise using the weather patterns and the cost of financing. Advanced risk and investment management tools includes the manage risks within enterprise; manage risks external to enterprise - climate change and weather patterns, financial conditions; and emissions and reporting.

\section{CONCLUSION}

Asset management describes the intended maintenance, asset replacement and contingency plans for each asset, and provides detail on the expenditure associated with each of these plans. Aging assets, uncertainty in load demand profile and renewable energy resources, and demand management create a challenge for the optimal maintenance and operation of electrical grid. This paper addresses the challenges and opportunities to improve the smart grid asset management and maintenance. This paper also presents the asset replacement alternatives. This paper discussed various issues of smart grid asset management in terms of various functions, importance, pros and cons relative to it. It also discusses various software tools available in the market and their functionality, challenges and improvements required to match the grid standards and expectations. The available tools are very advanced and perform almost all desirable functions, but it still requires improvements to make the future next generation grid.

\section{ACKNOWLEDGEMENTS}

This research work has been carried out based on the support of "Woosong University's Academic Research Funding - 2019”.

\section{REFERENCES}

[1] A. Carvallo and J. Cooper, "The Advanced Smart Grid: Edge Power Driving Sustainability,” Artech House, 2011.

[2] R. Habachi, et al., "Solving economic dispatch and unit commitment problem in smart grid system using eagle strategy based crow search algorithm," Indonesian Journal of Electrical Engineering and Computer Science, vol/issue: 14(3), pp. 1087-1096, Jun 2019.

[3] J. Ekanayake, et al., "Smart Grid: Technology and Applications," Wiley, Feb 2012.

[4] J. Yang, et al., "Dynamic Network Rating for Low Carbon Distribution Network Operation-A U.K. Application," IEEE Transactions on Smart Grid, vol/issue: 6(2), pp. 988-998, 2015.

[5] V. Aravinthan and W. Jewell, "Controlled Electric Vehicle Charging for Mitigating Impacts on Distribution Assets," IEEE Transactions on Smart Grid, vol/issue: 6(2), pp. 999-1009, 2015.

[6] O. K. Siirto, et al., "Optimal Distribution Network Automation Considering Earth Fault Events," IEEE Transactions on Smart Grid, vol/issue: 6(2), pp. 1010-1018, 2015.

[7] H. Ma, et al., "Smart Transformer for Smart Grid-Intelligent Framework and Techniques for Power Transformer Asset Management," IEEE Transactions on Smart Grid, vol/issue: 6(2), pp. 1026-1034, 2015.

[8] H. Wang, et al., "Research on Multi-objective Group Decision making in Condition-based Maintenance for Transmission and Transformation Equipment Based on D-S Evidence Theory," IEEE Transactions Smart Grid, vol/issue: 6(2), pp. 1035-1045, 2015.

[9] X. Liu, et al., "Risk Assessment in Extreme Events Considering the Reliability of Protective System," IEEE Transactions on Smart Grid, vol/issue: 6(2), pp. 1073-1081, 2015.

[10] S. M. Kaplan and F. Sissine, "Smart Grid: Modernizing Electric Power Transmission and Distribution; Energy Independence, Storage and Security; Energy Independence and Security Act and Resiliency; Integra (Government)," TheCapitol.Net, Inc., Sept 2009.

[11] R. P. Y. Mehairjan, et al., "Statistical life data analysis for electricity distribution cable assets - An Asset Management approach," IET and IAM Asset Management Conference, London, pp. 1-6, 2011.

[12] M. Shahidehpour and R. Ferrero, "Time management for assets: chronological strategies for power system asset management," IEEE Power and Energy Magazine, vol/issue: 3(3), pp. 32-38, 2005.

[13] J. A. Momoh, "Smart Grid: Fundamentals of Design and Analysis," Wiley-IEEE Press, Mar 2012.

[14] M. Cheng, et al., "Study on the model of advanced asset management in smart grid," Proc. of Electric Utility Deregulation and Restructuring and Power Technologies, Weihai, Shandong, pp. 781-785, 2011. 
[15] “Smart Grid Maturity Model,” Soft Engineering Institute, Carnegie Mellon, Technical Report, Sep 2011.

[16] K. A. Achmad, et al., "Smart City Readiness based on Smart City Council's Readiness Framework," International Journal of Electrical and Computer Engineering (IJECE), vol/issue: 8(1), pp. 271-279, Feb 2018.

[17] http://www.ngusummitna.com/media/whitepapers/Gridnet_NGUNA.pdf

[18] J. Lee, et al., "Data Analysis for Solar Energy Generation in a University Microgrid," International Journal of Electrical and Computer Engineering (IJECE), vol/issue: 8(3), pp. 1324-1330, Jun 2018.

[19] http://news.thomasnet.com/fullstory/Asset-Management-Platform-promotes-smart-grid-optimization-601763

[20] http://www-07.ibm.com/in/research/smartes_systems_research.html

[21] S. S. Kumar and P. A. D. V. Raj, "Fuzzy Logic based Stability Index Power System Voltage Stability Enhancement," International Journal of Computer and Electrical Engineering, vol/issue: 2(1), pp. 24-31, 2010.

[22] http://www.oracle.com/us/corporate/press/018637

[23] http://gridiantcorp.com/docs/Gridiant_Brochure.pdf

[24] N. A. B. Zainal, et al., "Gateway sink placement for sensor node grid distribution in LoRa smart city networks," Indonesian Journal of Electrical Engineering and Computer Science, vol/issue: 14(2), pp. 834-842, May 2019.

[25] http://www.energiamayorista.com.co/memorias_SG/fabricantes/siemens.pdf

[26] J. Meyers, "Preparing for the Future: How Asset Management Will Evolve in the Age of Smart Grid," White Paper, Schneider Electric, Jun 2012.

[27] “IEC Smart Grid Standardization Roadmap," Prepared by SMB Smart Grid Strategic Group (SG3), Jun 2010.

[28] M. I. A. Arafa and E. S. S. A. Said, "A different vision for uninterruptible load using hybrid solar-grid energy," International Journal of Power Electronics and Drive System (IJPEDS), vol/issue: 10(1), pp. 381-387, Mar 2019.

[29] M. McGranaghan, et al., "Renewable Systems Interconnection Study: Advanced Grid Planning and Operations," SANDIA Report, SAND2008-0944P Unlimited Release, Feb 2008.

[30] L. Rosyidi and K. Umam, "Design of Consumer Participative Device for Smart Grid Initiative," Proceeding of International Conference on Electrical Engineering, Computer Science and Informatics, Yogyakarta, Indonesia, Aug 2014. 